\title{
Total Mesorectal Excision, Lateral Lymphadenectomy and Autonomic Nerve Preservation for Lower Rectal Cancer: Significance in the Long-term Follow-up Study
}

\author{
KAZUO SHIROUZU, YUTAKA OGATA, YASUMI ARAKI, TERUO SASATOMI, \\ YASUHIRO NOZOE, MOTONORI NAKAGAWA AND KEIKO MATONO \\ Department of Surgery, Kurume University School of Medicine, \\ Kurume 830-0011, Japan
}

\begin{abstract}
Summary: We clarify the significance of total mesorectal excision (TME), lateral lymphadenectomy (LLA), and of autonomic nerve preservation (ANP) compared to conventional surgery (CVS), for lower rectal cancer. All 458 patients curatively resected between 1962 and 1997 were retrospectively investigated. In Period I from 1962-1974, when CVS only was performed, in Period II from 1975-1984, TME or TME+LLA was performed, and in Period III from 1985-1997, TME+ANP, TME+ANP+LLA, or TME+LLA was performed. In Dukes A+B disease, there was no significant difference among the three periods, regardless of operation methods. In Dukes $C$ disease, in Period I, CVS (42 patients: pts) had a local recurrence (LR) rate of $45.2 \%$ and 5 -year disease-free survival (5YDFS) rate of $33.3 \%$. In Period II, TME+LLA (82 pts) had a lower LR rate of $26.8 \%$ $(p=0.0628)$ and higher 5 YDFS $51.0 \%(p<0.05)$ vs CVS. In Period III, TME+ANP (12 pts) had LR $25.0 \%$ and 5 YDFS $55.6 \%$, TME+ ANP+LLA (45 pts) had LR $13.3 \%(p<0.005$, vs CVS) and 5YDFS 56.1\% ( $p<0.01$, vs CVS), and TME+LLA (18 pts) had LR $16.7 \%(p<0.05$, vs CVS) and 5 YDFS $20.8 \%$. Also, CVS had the lowest curability rate $64.8 \%$ and the highest mortality rate $7.2 \%$. TME and/or LLA was significant for reducing LR and improving survival in patients with Dukes C lower rectal cancer, compared to CVS. ANP was beneficial with LLA. TME+ANP was suitable for Dukes A or B disease.
\end{abstract}

Key words rectal cancer, total mesorectal excision, autonomic nerve preservation, lateral lymphadenectomy, disease-free survival, local recurrence

\section{INTRODUCTION}

The history of lymph node (LN) dissection for rectal cancer goes starts from the 1940's. It was then that LN metastasis was first conceived as a cause for a poor 5-year survival rate in lower rectal cancer below the peritoneal reflection [1,2]. In 1951, Sauer [3] proposed the clinical significance of lateral lymphatic spread as the reason for the poor survival rate in lower rectal cancer, and the need for $\mathrm{LN}$ dissection was further developed by adding higher ligation of the inferior mesenteric artery, as suggested by Ault [4,5]. After Miles' original procedure [6] of selective resection resulted in a poorer prognosis, Sauer and
Bacon [7] consequently advocated complete radical dissection of all lymph nodes off the iliac vessels. Sterns and Deddish [8] performed abdominopelvic LN dissection, and reported some improvement in survival for patients with advanced Dukes $\mathrm{C}$ disease compared with standard resection. However, they concluded that there was no general justification for their abdominopelvic LN dissection, because of the high complication rate, blood loss and long hospitalization time. In 1968, Harvey and Auchincloss [9] concluded that curative resection was unlikely to be achieved for any patient with Dukes $\mathrm{C}$ metastatic LNs distant from the primary tumor. In 1985, Glass et al. [10] retrospectively reviewed their experience 
with a more extended abdominoiliac lymphadenectomy. Neither improvement in survival nor decrease in local (or pelvic) recurrence (LR) could be demonstrated, and this attributed to their small number of 55 patients. In 1986, Enker [11] reported that the 5 -year survival rate was significantly improved in patients with Dukes C disease who had undergone en bloc pelvic lymphadenectomy, but LR was not reduced even though by the extended surgery. Therefore, many surgeons in Western countries tended to renounce extended abdominopelvic LN dissection, and moved toward favoring total mesorectal excision (TME), first reported in 1986 by Heald [12]. TME began to show a surprising improvement in survival and decrease in LR. In the following years, TME gradually replaced the use of extended pelvic lymphadenectomy in many countries. However, in Japan, the continued high LR was attributed to not only inadequate LN dissection but also an insufficient distal or lateral resection margin. Therefore, lateral lymphadenectomy (LLA) (sometimes termed pelvic lymphadenectomy or extended surgery) was performed between the early 1970s and the mid 1980s. The significance and benefit of LLA were debated and supported for a long time in Japan, and even the colorectal specialists at the National Cancer Center Hospital of Japan reported the significance of LLA to improve the survival rate and decrease LR [13-16]. This surgical technique was therefore widely practiced in Japan with the aim of ultimate radical cure. However, disadvantages such as urinary dysfunction or sexual functional disorder afflicted many patients following LLA [14], because LLA resected the mesorectum so widely and sacrificed the autonomic nerves in the radical resection to ensure all positive cancer cells were removed. LLA was thought to be a necessary and indispensable means to achieve curability. LLA demonstrated more excellent results compared to TME judging from the view point of a radical cure, but with respect to functional preservation, it was unavoidably inferior to TME. Around the mid-1980's, autonomic nerve preservation (ANP) was instituted alongside LLA to try to avoid postoperative functional disorders. This remarkable development of ANP with LLA was accomplished mainly in Japan. During this time, the ideology of functional preservation appeared to move in two different directions; to TME in Europe and to ANP in Japan. The compatibility of ANP with LLA has not been fully accepted, and is still being discussed even in Japan. Even advantages of LLA are still being questioned in Western countries. This is because lateral lymph node (LLN) metastasis does not occur so frequently and also because lateral tumor spread is a systemic disease. In the present study, we clarify the significance of TME, LLA, and ANP for lower rectal cancer, drawing on the very detailed clinical evidence we have recorded systematically during the long-term.

\section{PATIENTS AND METHODS}

Between 1962 and 1997, 1122 patients have undergone surgery for a rectal cancer. They have included 662 patients with advanced lower rectal cancer, of which 458 patients survived curative surgery. The detailed clinical data from the long-term follow-up of these 458 patients was retrospectively investigated here. The follow-up duration was divided into three periods; Period I ('62-'74), Period II ('75-'84) and Period III ( 85- 97), because the operation method was different in each period. The operation method and the Dukes stage according to each period are shown in Table 1. Both clinical and histological detailed information were registered into our computer, which was set up in 1982 using the database (dBASE III software, Ashton-Tate, Torrance, CA). Since 1982, both clinical information and histological findings of resected specimens have been prospectively registered in detail into the database [17-20]. Before 1982, those data were retrospectively registered by reviewing the medical records and available histological findings. Since 1995, we have been using the Visual dBASE software (Borland dBASE version 5.6J, CA) in the Windows Operating System (Microsoft, USA).

Follow-up investigation was carried out by outpatient visits, by letter, and by telephone, and the most recent date of contact was regarded as the final date of confirmation in each case. For the purposes of this study, the overall final follow-up date was the last day of February, 2001. Patients received postoperative protocol surveillance including tumor marker measurement, chest radiography and ultrasonography every month for the first year, every 3-to-6 months for the next two, three and four years, and then annually. Computed tomography (CT) or magnetic resonance imaging (MRI) was performed every 6 months for the first year, then annually.

A total of $15(3.3 \%)$ of the 458 patients had been lost from the follow-up by the final follow-up date, but 14 of these 15 patients had been followed for at least 8 years. The overall follow-up rate for more than 8 years was $99.8 \%$. The presence or absence of 
TABLE 1.

Operation methods and number of patients according to period and Dukes stage

\begin{tabular}{|c|c|c|c|c|c|c|}
\hline & \multirow{3}{*}{$\operatorname{CVS}(+)$} & \multicolumn{4}{|c|}{ TME $(+)$} & \multirow{3}{*}{ Total } \\
\hline & & \multicolumn{2}{|c|}{$\operatorname{ANP}(-)$} & \multicolumn{2}{|c|}{$\operatorname{ANP}(+)$} & \\
\hline & & $\operatorname{LLA}(-)$ & $\operatorname{LLA}(+)$ & $\operatorname{LLA}(-)$ & $\operatorname{LLA}(+)$ & \\
\hline \multicolumn{7}{|l|}{ Period I } \\
\hline Dukes A+B & 35 & & & & & 35 \\
\hline Dukes C & 42 & & & & & 42 \\
\hline \multicolumn{7}{|l|}{ Period II } \\
\hline Dukes A+B & & 24 & 79 & & & 103 \\
\hline Dukes C & & 9 & 82 & & & 91 \\
\hline \multicolumn{7}{|l|}{ Period III } \\
\hline Dukes A+B & & & 22 & 33 & 57 & 112 \\
\hline Dukes C & & & 18 & 12 & 45 & 75 \\
\hline Total & 77 & 33 & 201 & 45 & 102 & 458 \\
\hline
\end{tabular}

Period I : 1962-1974; Period II : 1975-1984; Period III : 1985-1997

CVS: conventional surgery; TME: total mesorectal excision; ANP: autonomic nerve preservation; LLA : lateral lymphadenectomy; $(+)$ : performed; $(-)$ : not performed

recurrence was determined by digital examination, barium enema, measurement of serum tumor marker level, and by findings on chest radiography, ultrasound, CT, and MRI. The site of recurrence was recorded for each patient and defined as local (or pelvic) recurrence, distant metastasis (liver, lung, bone or cerebrum) or peritoneal dissemination.

As to statistical analysis, the chi-square and the Fisher's exact tests were used to determine the statistical significance of any difference, and the Kaplan-Meier method [21] was used to calculate survival rates. Any significant difference in the survival rate was assessed using the log rank test (according to Peto [22]). The level of significance was defined at $\mathrm{p}<0.05$.

\section{RESULTS}

\section{Period I , 1962-1974}

We could review the medical records and histological findings in detail because they were relatively well-preserved. The inferior mesenteric artery (IMA) was not ligated at the origin of the aorta, but the superior rectal artery was excised as far distal as possible from the tumor, as described by Miles [7]. Neither anatomical lymphadenectomy, total mesorectal excision (TME) nor lateral lymphadenectomy (LLA) was performed. The operation was defined as conventional surgery (CVS) which was similar to that named by Enker [23]. So, not only the LLNs but also the upward LNs were not aggressively dissected. The mesorectum was bluntly dissected using hand maneuvers, as described by Miles [7]. Sometimes massive bleeding occurred from injury to the presacral vein or large vessels. The circumferential resection margin of the mesorectum including the distal edge, anterior, posterior and lateral wall of the rectum was not sufficiently isolated. The autonomic nerve plexus was never identified. Forty-one patients survived non-curative surgery, and 77 patients survived the curative surgery including 75 Miles' operations, 1 low anterior resection and 1 pullthrough method. Ten patients (6 curative, 4 noncurative) did not survive the operation. The curative operation rate was the lowest $64.8 \%(83 / 128)\left(\chi^{2}\right.$ $=10.4381 ; \mathrm{p}=0.0054)$ and the mortality rate (including hospital death at more than 30 days after operation) was the highest $7.2 \%(10 / 128) \quad\left(\chi^{2}=6.6910\right.$; $\mathrm{p}=0.0352$ ), compared to those in Period II and Period III. Subsequently, 37 patients died of cancer recurrence, after a median survival of 27 months (range 392 months). Twenty-five patients died of another disease, including 7 patients who died of old age, 3 of cardiovascular disease, 2 of cerebral disease, 1 of respiratory disease, 5 of cancer other than colon cancer, 1 of renal failure, 2 of hepatic disease, 1 of accident, and 3 of other disease. The median survival of these 25 patients had been 122 months (range 4-245 months). Eight patients are still alive, after a median follow-up of 342 months (range 324-453 months). Seven patients had been lost from the follow-up, but 
TABLE 2

Postoperative recurrence according to period and operation method

\begin{tabular}{|c|c|c|c|c|c|c|c|c|c|c|}
\hline & & LR & $\mathrm{LR}+\mathrm{DM}$ & DM & Others & Unknown & LR rate & \multicolumn{3}{|c|}{ DM rate } \\
\hline \multicolumn{11}{|l|}{ Period I } \\
\hline \multicolumn{11}{|l|}{ Dukes A+B } \\
\hline CVS & $(n=35)$ & 1 & 1 & 4 & 0 & 1 & $5.7 \%$ & \multicolumn{3}{|c|}{$14.3 \%$} \\
\hline \multicolumn{11}{|l|}{ Dukes C } \\
\hline CVS & $(n=42)$ & 10 & 9 & 8 & 0 & 3 & $45.2 \%^{\mathrm{a}}$ & \multicolumn{3}{|c|}{$40.5 \%$} \\
\hline \multicolumn{11}{|l|}{ Period II } \\
\hline \multicolumn{11}{|l|}{ Dukes $A+B$} \\
\hline TME & $(n=24)$ & 1 & 0 & 2 & 1 & 0 & $4.2 \%$ & \multirow{2}{*}{ NS } & $8.3 \%$ & \multirow{2}{*}{ NS } \\
\hline TME+LLA & $(n=79)$ & 2 & 2 & 9 & 0 & 2 & $5.1 \%$ & & $13.9 \%$ & \\
\hline \multicolumn{11}{|l|}{ Dukes C } \\
\hline TME & $(n=9)$ & 1 & 0 & 3 & 0 & 0 & $11.1 \%^{\mathrm{b}}$ & \multirow{2}{*}{ NS } & $33.3 \%$ & \multirow{2}{*}{ NS } \\
\hline TME+LLA & $(n=82)$ & 12 & 10 & 17 & 3 & 1 & $26.8 \% \mathrm{c}$ & & $32.9 \%$ & \\
\hline \multicolumn{11}{|l|}{ Period III } \\
\hline \multicolumn{11}{|l|}{ Dukes A+B } \\
\hline TME+ANP & $(n=33)$ & 1 & 1 & 3 & 2 & 0 & $6.1 \%$ & \multirow{3}{*}{ NS } & $12.1 \%$ & \multirow{3}{*}{$\int \mathrm{NS}$} \\
\hline TME+ANP+LLA & $(n=57)$ & 1 & 0 & 5 & 0 & 0 & $1.8 \%$ & & $8.8 \%$ & \\
\hline TME+LLA & $(n=22)$ & 2 & 0 & 3 & 2 & 0 & $9.1 \%$ & & $13.6 \%$ & \\
\hline \multicolumn{11}{|l|}{ Dukes C } \\
\hline $\mathrm{TME}+\mathrm{ANP}$ & $(n=12)$ & 1 & 2 & 1 & 1 & 0 & $25.0 \% \mathrm{~d}$ & \multirow{3}{*}{ NS } & $25.0 \%$ & \multirow{3}{*}{ NS } \\
\hline TME+ANP+LLA & $(n=45)$ & 5 & 1 & 14 & 0 & 0 & $13.3 \%^{\mathrm{e}}$ & & $33.3 \%$ & \\
\hline TME+LLA & $(n=18)$ & 1 & 2 & 10 & 0 & 1 & $16.7 \%^{\mathrm{f}}$ & & $66.7 \%$ & \\
\hline
\end{tabular}

LR: local (pelvic) recurrence; DM: distant metastasis including liver, lung, cerebrum, and bone metastasis; Others: peritoneal seeding and inguinal nodes metastasis; CVS: conventional surgery; TME: total mesorectal excision; LLA: lateral lymphadenectomy; ANP: autonomic nerve preservation; As to Dukes A+B, there was no significant difference concerning local recurrence regardless of operation methods through the three periods. a vs b: not significant (NS); a vs c: NS ( $p=0.0628$ ); a vs d: NS; a vs e: $p<0.005$; a vs $f: p<0.05$; b vs c: NS; $d$ vs e: NS; $d$ vs $f$ : NS; e vs $f$ : NS

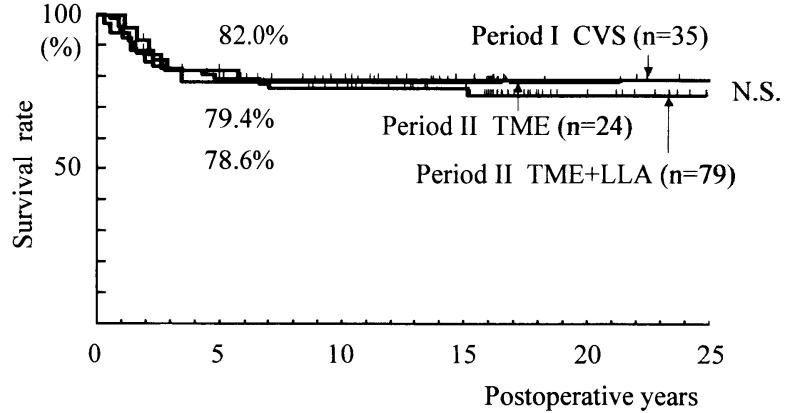

Fig. 1. Disease-free survival in Dukes A+B disease patients during Period I and Period II. No significant difference was noted among the three groups.

they had been followed for at least 8 years, with a median follow-up of 184 months (range 100-268 months).

Local recurrence ( $\boldsymbol{L R})$ : As shown in Table 2, in patients with Dukes A+B disease (35 patients), and Dukes C disease (42 patients), the LR rate was $5.7 \%$, and $45.2 \%$, respectively.

Distant metastasis (DM): As shown in Table 2 , in patients with Dukes A+B disease, and Dukes C disease, the DM rate was $14.3 \%$, and $40.5 \%$, respectively.

Survival: The 5-year disease-free survival (5YDFS) rate in patients with Dukes A+B disease, and with Dukes C disease was $82.0 \%$ (Fig. 1), and $33.3 \%$ (Fig. 2), respectively.

\section{Period II, 1975-1984}

During this period, aorto-caval and LLA was almost routinely performed for advanced lower rectal cancer $(83 \%$; 161/194). The IMA was ligated at the origin of the aorta. The aorto-caval lymphadenectomy included not only the LNs in front of the aorta and caval vein but also the para-aortic LNs that existed on both sides of those vessels. The height of the lymphadenectomy was from the lower edge of the duodenum and left renal vein down to the aortic bifurcation. The LLA included the LLNs [internal, external and common iliac vessels region LNs 
(No272, No293 and No273), the obturator space region LNs (No282), and the middle rectal artery region LNs (No262)], as classified by the Japanese Rules for the Treatment of Colorectal Cancer [24]. Bilateral LLA was usually performed for an advanced lower rectal cancer. However, when the tumor involved less than half the rectum and was confined to the lateral side, only iso-lateral LLA was performed, and contra-lateral LLA was not. The autonomic nerve plexus including hypogastric and pelvic plexus were routinely not preserved but sacrificed. So, not only the upward LNs but also the aorto-caval LNs and LLNs were sufficiently dissected. The circumferential resection margin of the mesorectum including the distal edge, anterior Denovillier's fascia, posterior Waldeyer's fascia and lateral ligament were completely removed by sharp dissection using an electric-knife and scissors under direct vision, which was consistent with TME, as reported by Heald and Ryall [12].

Eighty-six patients survived non-curative surgery, and 194 patients survived curative surgery including 24 low anterior resections, 11 ultra low anterior resections, 10 pull-through methods, 145 abdominoperineal resections, and 4 total pelvic exenterations. Ten patients (4 curative, 6 non-curative) did not survive the operation. The curative operation and mortality rates (including hospital death at more than 30 days after operation) were $68.3 \%(198 / 290)$ and $3.4 \%$ (10/290), respectively. As shown in Table 1, 33 of these patients had TME without LLA (TME Group; Dukes A+B: 24, Dukes C: 9). TME with aorto-caval and lateral lymphadenectomy was performed in 161 patients (TME+LLA Group; Dukes A+B: 79, Dukes C: 82). Sixty-eight patients died of cancer recurrence after a median survival of 35 months (range 13-201 months). Seventy-four patients died of another disease, including 16 patients who died of old age, 9 of cardiovascular disease, 11 of cerebral disease, 8 of respiratory disease, 14 of cancer other than colon cancer, 3 of hepatic disease, 1 of renal failure, 1 of ileus, 1 of gastrointestinal bleeding, 3 of radiation enteritis, 1 of marine accident, and 6 of other disease, after a median survival of 142 months (range 6-300 months). Forty-seven patients are still alive, after a median follow-up of 211 months (range 129-307 months). Five patients had been lost from the followup, but they had been followed for at least 10 years with a median follow-up of 154 months (range 129192 months).

$\boldsymbol{L R}$ : As shown in Table 2, in patients with Dukes $\mathrm{A}+\mathrm{B}$ disease, the LR rate was $4.2 \%$ in the TME
Group (24 patients), and $5.1 \%$ in the TME+LLA Group (79 patients). No significant difference was noted either between the two groups or between each group and the Period I CVS Group.

In patients with Dukes $\mathrm{C}$ disease, LR occurred at a rate of $11.1 \%$ in the TME Group ( 9 patients), and $26.8 \%$ in the TME+LLA Group (82 patients). No significant difference was noted between the two groups, or between the TME Group and the CVS Group, because of the small number of TME patients. However, the TME+LLA Group tended to have a LR rate $(\mathrm{p}=0.0628)$ lower than that in the Period I CVS Group.

$\boldsymbol{D M}$ : As shown in Table 2, in patients with Dukes $\mathrm{A}+\mathrm{B}$ disease, the DM rate was $8.3 \%$ in the TME group, and $13.9 \%$ in the TME+LLA Group. No significant difference was noted either between the two groups or between each group and the Period I CVS Group.

In patients with Dukes $\mathrm{C}$ disease, DM occurred at a rate of $33.3 \%$ in the TME Group and $32.9 \%$ in the TME+LLA Group. No significant difference was noted between the two groups or between each group and the Period I CVS Group.

Survival: In patients with Dukes A $+B$ disease, the 5YDFS rate in the TME Group, and in the TME+ LLA Group was $78.6 \%$, and $79.4 \%$, respectively (Fig. 1). No significant difference was noted either between the two groups or between each group and the Period I CVS Group.

In patients with Dukes $\mathrm{C}$ disease, the 5YDFS rates in the TME Group and in the TME+LLA Group was $57.1 \%$ and $51.0 \%$, respectively (Fig. 2).

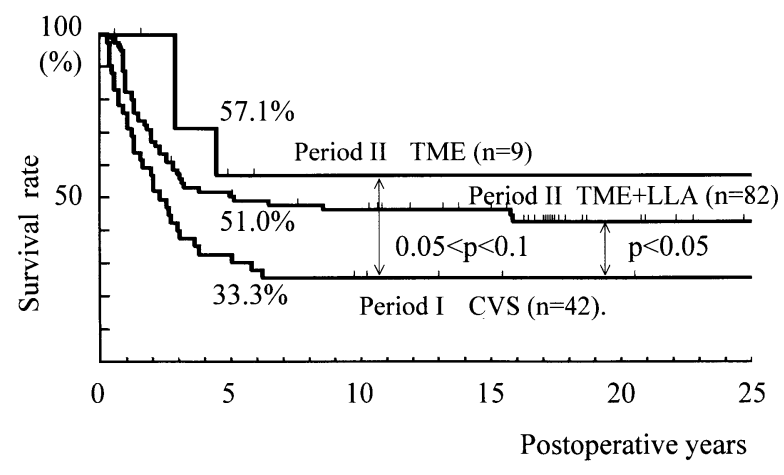

Fig. 2. Disease-free survival in Dukes $\mathrm{C}$ disease patients during Period I and Period II. A significant difference was noted between the Period II TME + LLA Group and Period I CVS Group $(\mathrm{p}<0.05)$. There was a trend toward higher disease-free survival rate in the Period II TME than that in the Period I CVS Group $(0.05<\mathrm{p}<0.1)$. 
No significant difference was noted between the two groups. The TME group tended to show a higher disease-free survival rate $\left(\chi^{2}=3.2290 ; 0.05<\mathrm{p}<0.1\right)$ than the Period I CVS Group, but no significant difference was noted because of the small number of 9 patients in the TME Group. However, the 5YDFS rate in the TME+LLA Group was significantly higher $\left(\chi^{2}=5.0385 ; p<0.05\right)$ than that in the Period I CVS Group (Fig. 2).

\section{Period III, 1985-1997}

While aorto-caval lymphadenectomy had usually been performed in Period II, it was not usually performed in Period III. So, para-aortic lymphadenectomy was not routinely performed, except for unusual cases. In Period III, the IMA was ligated at the origin of the aorta. Pelvic lymphadenectomy was enforced and the mesorectum was completely excised by techniques similar to those in Period II. So, not only the upward LNs but also the LLNs were sufficiently dissected. The autonomic nerve plexus including hypogastric and pelvic plexus were completely preserved, as far as possible. When tumor invasion was suspected against the adjacent nerve plexus, then the autonomic nerve plexus were removed to avoid a risk of residual cancer cells. ANP was employed for most patients with rectal cancer (147/187: 78.6\%). Fifty-one patients survived noncurative surgery, and 187 patients survived curative surgery including 42 low anterior resections, 40 ultra low anterior resections, 100 abdominoperineal resections, and 5 total pelvic exenterations. Six patients (5 curative, 1 non-curative) did not survive the operation. The curative operation and mortality rates (including hospital death at more than 30 days after operation) were $78.7 \%$ (192/244) and 2.5\% (6/244), respectively. As shown in Table 1, 45 of 187 patients had TME+ANP (Dukes A+B: 33, Dukes C: 12), and 102 patients had TME+LLA+ANP (Dukes A+ B: 57, Dukes C: 45), while the other 40 patients had TME+LLA without ANP because cancer cells were suspected to have invaded the autonomic nerve tissue (TME+LLA Group; Dukes A+B: 22, Dukes C: 18), so we could not preserve the autonomic nerve plexus. This situation was similar to that in the TME+LLA Group in Period II. Forty-five patients died of cancer recurrence after a median survival of 38 months (range 6-139 months). Thirty-three patients died of another disease, including 5 patients who died of old age, 5 of cardiovascular disease, 3 of cerebral disease, 4 of respiratory disease, 8 of cancer other than colon cancer, 3 of accident, 1 of ileus, and 4 of other dis- ease, after a median survival of 54 months (range 6164 months). One hundred and six patients are still alive, with a median follow-up of 102 months (range 42-185 months). Three patients had been lost from the follow-up, but they had been followed for at least 8 years (except for one patient followed for only 27 months), with an overall median follow-up of 91 months (range 27-155 months).

$\boldsymbol{L R}$ : As shown in Table 2, in patients with Dukes $\mathrm{A}+\mathrm{B}$ disease, the LR rate was $6.1 \%$ in the TME+ ANP Group (33 patients), $1.8 \%$ in the TME+ANP+ LLA Group (57 patients), and $9.1 \%$ in the TME+ LLA Group (22 patients). No significant difference was noted either among the three groups or between each group and Period I CVS Group.

In patients with Dukes C disease, LR occurred at a rate of $25 \%$ in the TME+ANP Group (12 patients), $13.3 \%$ in the TME+ANP+LLA Group (45 patients) and $16.7 \%$ in the TME+LLA Group (18 patients). No significant difference was noted among the three groups. However, the LR rates in the TME+ANP+ LLA and TME+LLA groups were each significantly lower $\left(\chi^{2}=9.2966 ; p<0.005\right.$, Fisher's exact test; $p<$ 0.05) than that in the Period I CVS Group.

$\boldsymbol{D M}$ : As shown in Table 2, in patients with Dukes $\mathrm{A}+\mathrm{B}$ disease, the DM rate was $12.1 \%$ in the TME+ ANP Group, $8.8 \%$ in the TME+ANP+LLA Group, and $13.6 \%$ in the TME+LLA Group. No significant difference was noted either among the three groups or between each group and the Period I CVS Group.

In patients with Dukes $\mathrm{C}$ disease, DM occurred at a rate of $25 \%$ in the TME+ANP Group, $33.3 \%$ in the TME+ANP+LLA Group, and $66.7 \%$ in the TME+ LLA Group. No significant difference was noted either among the three groups or between each group and the Period I CVS Group.

\section{Survival in the patients who underwent}

$\boldsymbol{A N P}$ : In patients with Dukes A+B disease, the $5 Y$ YFS rate in the TME+ANP Group, and in the TME+ANP+ LLA Group was $77.8 \%$ and $90.1 \%$, respectively (Fig. 3). No significant difference was noted either between the two groups or between each group and the Period I CVS Group.

In patients with Dukes $C$ disease, the 5YDFS rate in the TME+ANP Group, TME+ANP+LLA Group was $55.6 \%$, and $56.1 \%$, respectively (Fig. 4). No significant difference was noted between the Period III TME+ANP Group and the Period I CVS Group, because of the small number of patients in the TME+ANP Group. However, a significant difference was noted between the TME+ANP+LLA Group and CVS Group $\left(\chi^{2}=6.7948 ; p<0.01\right)$. 


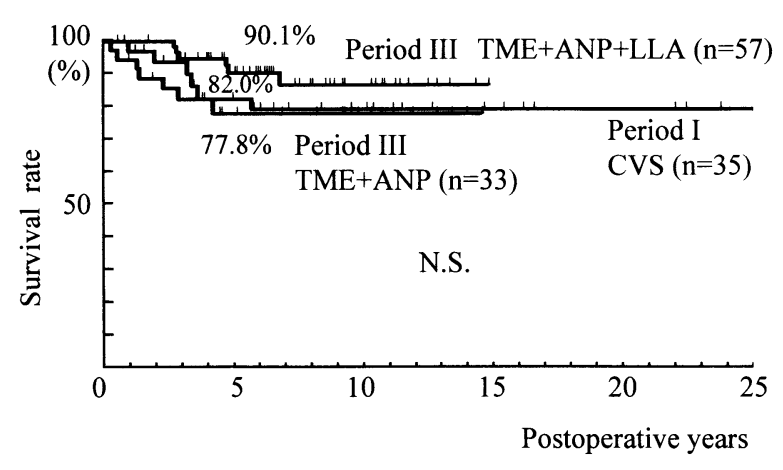

Fig. 3. Disease-free survival in Dukes A+B disease patients who underwent ANP during Period III. No significant difference was noted among the three groups, regardless of operation methods.

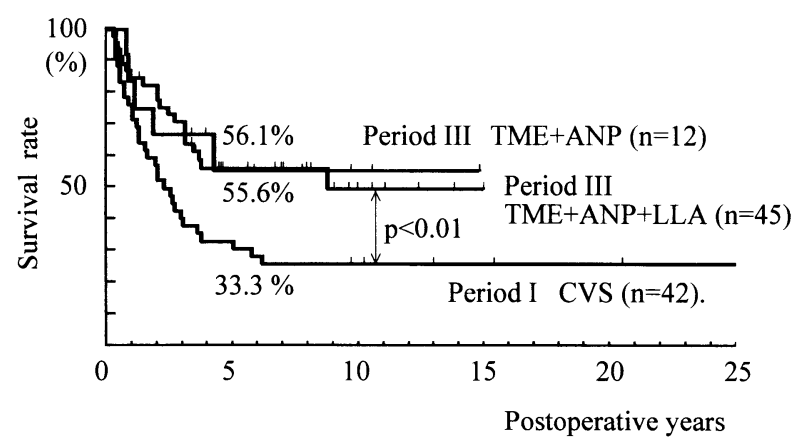

Fig. 4. Disease-free survival in Dukes C disease patients who underwent ANP during Period III. No significant difference was noted between the Period III TME+ANP Group and the Period I CVS Group, because of the small number of patients in the TME+ANP Group. However, a significant difference was noted between the Period III TME+ ANP+ LLA Group and the Period I CVS Group $(\mathrm{p}<0.01)$.

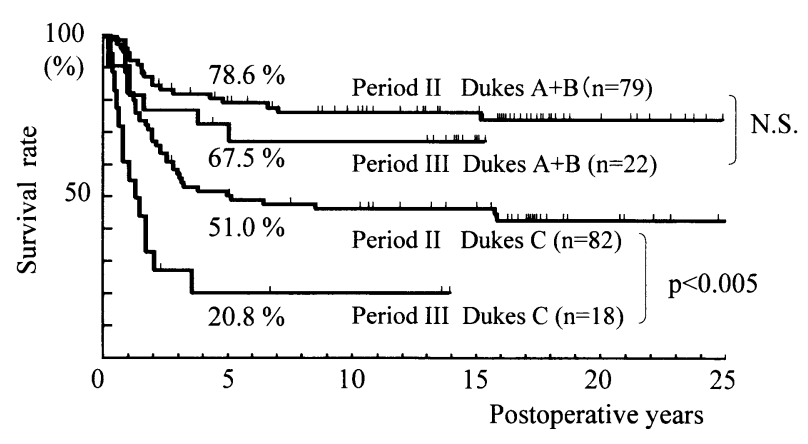

Fig. 5. Disease-free survival in patients who did not undergo ANP (TME+LLA) during Period II and Period III. No significant difference was noted in Dukes $\mathrm{A}+\mathrm{B}$ disease patients between Period II and Period III. However, a significant difference was noted in Dukes $\mathrm{C}$ disease patients between Period II and Period III $(\mathrm{p}<0.005)$.
Survival in the patients who did not undergo ANP (TME + LLA): As shown in Fig. 5, in patients with Period III Dukes A+B disease, the 5YDFS rate was $67.5 \%$. No significant difference was noted between this and the $78.6 \%$ in Period II Dukes $\mathrm{A}+\mathrm{B}$ disease. However, in patients with Dukes $\mathrm{C}$ disease, the 5YDFS rate in Period III showed a significantly lower rate of $20.8 \%\left(\chi^{2}=\right.$ 9.7396; $\mathrm{p}<0.005)$ compared to the $51.0 \%$ in Period II Dukes $\mathrm{C}$ disease.

\section{Incidence of lateral lymph node ( $L L N)$ metastasis}

LN metastasis was found in 208 (Dukes C disease; $45.4 \%$ ) of the 458 patients with lower rectal cancer who underwent curative surgery. LLA was performed in 303 of 458 patients. LLN metastasis was found in $47(22.6 \%)$ of the 208 patients with Dukes $\mathrm{C}$ disease, and was found in $47(15.5 \%)$ of the 303 patients who underwent LLA (Table 3).

As to the site of the LLN metastasis, No262 LN metastasis was detected in 21 patients (42\%), No282 $\mathrm{LN}$ in 15 patients (30\%), No272 LN in 11 patients (22\%), No273 LN in 3 patients (6\%), and No293 LN in 3 patients $(6 \%)$. Downward LN metastasis to inguinal nodes was also found in 7 patients (14\%).

Lateral and upward LN metastasis: As shown in Table 3, 37 (18.7\%) of the 198 patients had LLN metastasis accompanied with upward LN metastasis. Only $10(9.5 \%)$ of the 105 patients had LLN metastasis without upward LN metastasis. A slight correlation was noted between LLN and upward LN metastasis (Table 3, $\chi^{2}=3.7244 ; \mathrm{p}=0.053$ ).

LLN metastasis and size of the tumor: When the tumor size was less than $3 \mathrm{~cm}$, the LLN metastasis was found in only one $(5.6 \%)$ of 18 patients, and when the tumor size was $5 \mathrm{~cm}$ or more, LLN metastasis was found in 34 (19.3\%) of 176 patients, but no significant correlation was noted (Table 3 , $\chi^{2}=4.9934 ; \mathrm{p}=0.082$ ).

LLN metastasis and circumference of the tumor: When the tumor was less than half the circumference, LLN metastasis was found in $11(9.5 \%)$ of 116 patients, when the tumor was between $1 / 2-2 / 3$ of the circumference, LLN metastasis was found in $17(24.6 \%)$ of 69 patients, and when the tumor was more than 2/3, LLN metastasis was found in 19 $(16.1 \%)$ of 118 patients, with a significant correlation (Table 3, $\chi^{2}=7.6334 ; \mathrm{p}<0.05$ ).

LLN metastasis and depth of invasion: LLN metastasis was found in $8(13.1 \%)$ of 61 patients with proper muscle invasion (T2), in $32(15.3 \%)$ of 209 patients with invasion beyond the proper muscle (T3), 
TABLE 3.

Incidence of lateral lymph node metastasis $(L L N-m)$ in 303 patients who underwent lateral lymphadenectomy

\begin{tabular}{|c|c|c|c|c|c|}
\hline \multirow[b]{2}{*}{ Upward metastasis } & \multirow[b]{3}{*}{$(n=105)$} & \multirow{3}{*}{$\begin{array}{c}\begin{array}{c}\text { LLN-m } \\
\text { negative }\end{array} \\
95\end{array}$} & \multirow[t]{2}{*}{$\begin{array}{l}\text { LLN-m } \\
\text { positive }\end{array}$} & \multicolumn{2}{|c|}{$\begin{array}{c}\text { p-value } \\
\text { (chi-square test) }\end{array}$} \\
\hline & & & & $\mathrm{p}=0.053$ & $(2 \times 2)$ \\
\hline negative & & & $10(9.5)$ & & \\
\hline positive & $(n=198)$ & 161 & $37(18.7)$ & & \\
\hline Tumor size & & & & $\mathrm{p}=0.082$ & $(3 \times 2)$ \\
\hline$<3 \mathrm{~cm}$ & $(\mathrm{n}=18)$ & 17 & $1(5.6)$ & & \\
\hline $3-5 \mathrm{~cm}$ & $(n=109)$ & 97 & $12(11.0)$ & & \\
\hline$\geqq 5 \mathrm{~cm}$ & $(n=176)$ & 142 & $34(19.3)$ & & \\
\hline Circumference & & & & $\mathrm{p}<0.05$ & $(3 \times 2)$ \\
\hline$<1 / 2$ & $(n=116)$ & 105 & $11(9.5)$ & & \\
\hline $1 / 2-2 / 3$ & $(n=69)$ & 52 & $17(24.6)$ & & \\
\hline$\geqq 2 / 3$ & $(n=118)$ & 99 & $19(16.1)$ & & \\
\hline Depth of invasion & & & & $\mathrm{p}=0.579$ & $(3 \times 2)$ \\
\hline $\mathrm{T} 2$ & $(n=61)$ & 53 & $8(13.1)$ & & \\
\hline T3 & $(n=209)$ & 177 & $32(15.3)$ & & \\
\hline $\mathrm{T} 4$ & $(n=33)$ & 26 & $7(21.2)$ & & \\
\hline Histology & & & & $\mathrm{p}<0.05$ & $(3 \times 2)$ \\
\hline well & $(n=191)$ & 168 & $23(12.0)$ & & \\
\hline mode & $(n=80)$ & 65 & $15(18.8)$ & & \\
\hline others & $(n=32)$ & 23 & $9(28.1)$ & & \\
\hline Lymphatic permeation & & & & $\mathrm{p}<0.0001$ & $(3 \times 2)$ \\
\hline ly 0 & $(n=136)$ & 125 & $11(8.1)$ & & \\
\hline ly1 & $(n=93)$ & 81 & $12(12.9)$ & & \\
\hline $\operatorname{ly} 2+\operatorname{ly} 3$ & $(n=74)$ & 50 & $24(32.4)$ & & \\
\hline Venous invasion & & & & $\mathrm{p}<0.005$ & $(3 \times 2)$ \\
\hline $\mathrm{v} 0$ & $(\mathrm{n}=58)$ & 52 & $6(10.3)$ & & \\
\hline v1 & $(n=168)$ & 148 & $20(11.9)$ & & \\
\hline $\mathrm{v} 2+\mathrm{v} 3$ & $(n=77)$ & 56 & $21(27.3)$ & & \\
\hline
\end{tabular}

T2, T3 and T4 according to UICC TNM system

well: well differentiated adenocarcinoma; mode: moderately-differentiated adenocarcinoma; others: mucinous adenocarcinoma or poorly-differentiated adenocarcinoma; ly0, v0: negative; ly1,v1: slight; ly2, v2: moderate; ly3, v3: marked

and in $7(21.2 \%)$ of 33 patients with adjacent organ or visceral peritoneum invasion (T4), with no significant correlation (Table 3, $\chi^{2}=1.0920 ; \mathrm{p}=0.579$ ).

LLN metastasis and histology: When the tumor showed well-differentiated adenocarcinoma, moderately-differentiated adenocarcinoma or others (mucinous adenocarcinoma or poorly-differentiated adenocarcinoma), LLN metastasis was found in 23 $(12.0 \%)$ of 191 patients, in $15(18.8 \%)$ of 80 patients, and in $9(28.1 \%)$ of 32 patients, with a significant correlation (Table 3, $\chi^{2}=6.2794 ; \mathrm{p}<0.05$ ).

LLN metastasis and lymphatic permeation: As previously reported [19], the grade of lymphatic permeation was divided into four degrees; ly0: negative, ly1: slight, ly2: moderate, and ly3: marked). When the degree was ly0, ly 1 , and ly $2+$ ly 3 , LLN metastasis was found in $11(8.1 \%)$ of 136 patients, in $12(12.9 \%)$ of 93 patients, and in $24(32.4 \%)$ of 74 patients, with a significant correlation (Table $3, \chi^{2}=$ 22.2927; $<<0.0001$ ).

LLN metastasis and venous invasion: As previously reported [17], the grade of venous invasion was divided into four degrees; $\mathrm{v} 0$ : negative, $\mathrm{v} 1$ : slight, v2: moderate, v3: marked). When the degree was v0, v1, and v2+v3, LLN metastasis was found in $6(10.3 \%)$ of 58 patients, in $20(11.9 \%)$ of 168 patients, and in $21(27.3 \%)$ of 77 patients, with a significant correlation (Table $3, \chi^{2}=10.9762 ; \mathrm{p}<0.005$ ).

The number of LLNs with positive metastasis and survival: Thirty-two of 47 patients had cancer recurrence. For a recurrent tumor, hepatic resection, pulmonary resection, perineal tumor resection, or total pelvic exenteration was performed each in one patient, and palliative resection in 5 patients, radiation in 3 patients, and chemotherapy in all patients. The number of LLNs with positive metas- 


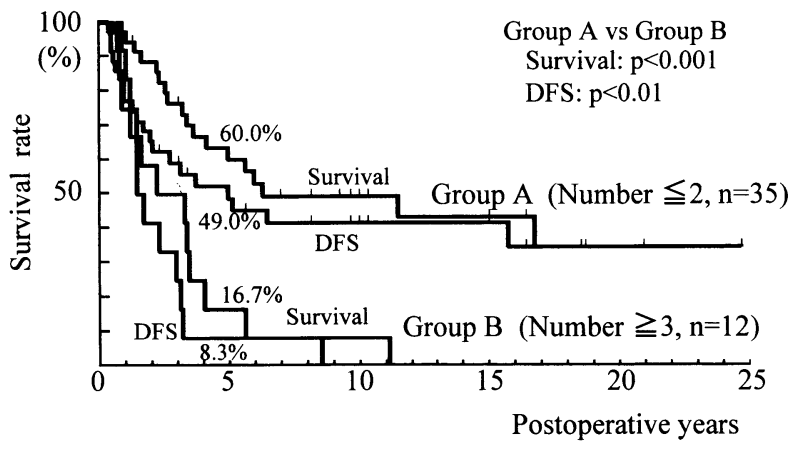

Fig. 6. Survival and disease-free survival (DFS) according to the number of lateral lymph nodes (LLNs) with positive metastasis. Patients were divided into two groups according to the number of LLNs with positive metastasis; Group A consisted of those with less than 3 , and the other Group B of those with 3 or more. There was no significant difference between the survival and DFS rates in each group. However, Group A had a significantly higher 5-year survival $(\mathrm{p}<0.001)$ and higher 5-year disease-free survival rates $(\mathrm{p}<0.01)$ than those in Group B.

tasis was less than 3 in 35 patients, and was 3 or more in 12 patients. In the former group (Group A), 18 of 35 patients died of cancer recurrence, including 6 patients who died of liver metastasis, 5 of lung metastasis, 2 of bone metastasis, 5 of local recurrence. Thirteen of 35 patients are still alive and disease-free, after a median follow-up of 119 months (range 40-295 months). Three patients had died of cancer-unrelated disease. One patient had been lost from the follow-up. In the latter group (Group B), all patients died of cancer recurrence, with a median survival of 32 (range 10-133 months).

The 5-year survival (5YS) and 5YDFS rates were $60.0 \%$ and $49.0 \%$ in the Group A, and $16.7 \%$ and $8.3 \%$ in the Group B (Fig. 6). In the Group A, the $5 Y$ Y rate improved slightly compared to the 5YDFS rate, with no significant difference. Also, the Group A had significantly higher 5YS $\left(\chi^{2}=12.8022 ; \mathrm{p}<\right.$ $0.001)$ and 5YDFS $\left(\chi^{2}=7.0315 ; \mathrm{p}<0.01\right)$ rates than those in the Group B.

\section{DISCUSSION}

Postoperative follow-up investigation is important to estimate correctly the final results from surgical treatment, as reported by Pietra [25]. The final appraisal is to know the survival, disease-free survival, and recurrence rates. Kurume University is located in Kurume City in the southern part of Japan, and is the center of the residential district in the city. Our university has a 70-year history since establishment. And now, we have many affiliated public or private hospitals like satellites around our university. Their doctors have introduced many cancer patients to our hospital. Also through their collaboration, this postoperative follow-up investigation could be successfully achieved. Fifteen patients $(3.3 \%)$ had been lost from the follow-up at the final follow-up date, but 14 of them had been followed for at least 8 years and were disease-free at the last contact. Our pathological study has been prospective since 1982, as previously reported by one of the authors (Shirouzu) [17-20]. Our present follow-up study provides support for reliable evidence-based medicine.

Over the past thirty years, a mountain of problems has been discussed about the treatment for rectal cancer patients. These problems include the definition and diagnosis of LLN metastasis, the significance of lymphadenectomy, indication for LLA, postoperative functional disorder (urinary or sexual disorder), survival benefit, and the need for randomized controlled trial, and so on. The definition of LLN is derived from the conception of lateral spread in rectal cancer. The lymphatic flow consists of upward spread streaming along the superior rectal artery and inferior mesenteric artery, lateral spread streaming along the middle rectal artery, internalexternal and common iliac artery, and extending up into the obturator space. Another lymphatic flow is known as downward spread streaming along the inferior rectal artery up to the inguinal LNs [26]. According to the lymphatic drainage anatomy and the high incidence of metastasis, the main LLN includes the middle rectal LNs, obturator LNs and iliac LNs (internal, external and common iliac nodes) $[16,27,28]$.

During the 1960s in Japan, colonic lymphadenectomy was performed with no anatomical knowledge of LN metastasis. Such narrow lymphadenectomy, termed conventional surgery (CVS), was applied for every patient. Extended aortopelvic lymphadenectomy including LLA was widely performed by many Japanese surgeons between the mid 1970s and the early 1980s [13-16]. In Western countries, the advantages from lymphadenectomy were gradually being reported, especially from LLA. Sterns and Deddish [8] tried to establish LLA during the 1950s, but could not find any clinical significance. In fact, either CVS, TME without ANP or TME+ANP seems to be adequate for patients with Dukes A or B disease as 
shown in our prognostic results (Figs 1 and 3). However, the recent concept of TME seems to completely remove the fatty tissue around the rectum with identifying autonomic nerves. So, TME+ANP would be an optimal surgical method for patients with Dukes A or B disease to avoid functional disorders, if LN metastasis had been correctly diagnosed before the operation.

On the other hand, the prognosis of Dukes $\mathrm{C}$ patients was poor with only CVS, and this was found in our study of the results (Figs 2 and 4). The LR and 5 YDFS rates in Dukes $\mathrm{C}$ patients during Period I were similar to those in some Western countries [29]. Also, the prognosis during Period II and Period III was significantly better than that in Period I. The significance of anatomical lymphadenectomy, in other words, TME or LLA, was demonstrated clearly in our study. Some other authors have also advocated the importance of TME and LLA [13-16,30,31]. On the other hand, Hida [32] reported some doubt as to the significance of LLA, because of the low survival rate and high rate of functional disorders. There are other reasons for their doubting the advantages of LLA. One of these reasons is that lateral tumor spread is a systemic disease, not a regional disease. Our results showed that the survival rate was extremely high in Dukes C patients during Period II and Period III compared to that in Dukes $\mathrm{C}$ patients during Period I. These results showed clearly that even patients with LLN metastases could be cured by LLA. If the LLA had not been performed during the Period II and Period III, LR could have occurred most likely at a high rate. Moreover, the 5-year survival rate in patients having a small number of LLN metastases was better, compared to that of patients with a large number of LLN metastases. The 5YDFS rate showed that recurrence did not always occur in patients having a small number of LLN metastasis, as shown in Fig. 6. Therefore, LLN spread is not always a systemic disease, when the number of metastasis is small. In such cases, survival rate could be more improved by multidisciplinary treatments including chemotherapy, immunotherapy, or radiotherapy. Secondly, it is well known that LLN metastasis does not occur so frequently. The incidence was $22.6 \%$ in Dukes $\mathrm{C}$ disease, and $15.5 \%$ in those patients who underwent LLA, similar to that described by other authors $[16,27,28]$. This seems to be not so low. Thirdly, LLA accompanies postoperative mortality, morbidity, long hospitalization and expensive medical treatment cost compared to TME. Actually, there are some differences in physique and constitution between Japanese and Western patients. Either obesity or blood hypercoagulability has a high risk to critical morbidity such as cardiovascular, lung or brain complications. However, Fabrizio [33] reported that wide pelvic lymphadenectomy did not increase the intraoperative or early postoperative complication rate, although a small number of 27 patients. Recently, the morbidity and mortality rates were extremely low, even though LLA was performed, as also reported by Moriya [16]. Many surgeons have reported that the mortality and morbidity were extremely low also in patients after TME $[34,35]$. On the other hand, Period I CVS had the lowest curability and the highest mortality, that might have been caused by ignorance of anatomical considerations, poor hand maneuvers in blunt and rough dissection, and poor perioperative management. Uncontrollable massive bleeding often happened. We must pay attention to the high mortality rate after CVS, as reported by Bolognese [36]. Overall LLA seems to be as safe as TME for most rectal cancer patients, at least for Japanese patients. Additionally, Japanese patients do not need to pay all the incurred medical treatment cost by themselves, because most of the medical treatment cost is covered by national health insurance. The load to patients of the medical treatment cost is lighter compared to that in some Western countries. This financial consideration might be at the root of the difference in philosophy concerning surgical methods including LLA.

The indication for LLA has also been controversial, even in Japan. To clarify the indication, there is nothing to depend upon except accumulated clinical and pathological information, and long-standing clinical experience. The important clinical indicator for LLA seems to be the circumference of the tumor. Of the many indicators, lymphatic permeation was found to be the most important histologic factor. We have already reported the important correlation between lymphatic permeation of preoperative punch biopsy and LN metastasis of rectal cancer [37]. These factors are often beneficial for some patients with a low risk indicator to avoid LLA. LLA would not be needed for patients in whom the tumor circumference is less than half the rectal wall circumference. Of course, when the tumor is located above the peritoneal reflection, it is not necessary to consider the lymphatic streams. On the other hand, when the tumor is located below the peritoneal reflection, the lymphatic streams are important for patients with advanced lower rectal cancer (T2, T3 
and T4 tumors in the UICC TNM system) [38]. Although only a handful of patients have LLN metastasis, LLA is unavoidable for some patients with advanced lower rectal cancer, because an accurate diagnosis for LLN metastasis cannot be made, even though using computed tomography and magnetic resonance imaging. Although Tada reported a unique and highly accurate diagnostic method for LLN metastasis using ultrasound [39], the accuracy is usually at $60-80 \%$ [40-42].

Recently, TME reported by Heald and Ryall [12] gave a surprising impact to many surgeons as an excellent technique to improve survival, decrease LR and preserve functional capabilities. This technique seems to have taken the place of LLA in many countries. Indeed, many Japanese surgeons also perform TME with sharp dissection under direct vision. This is a different technique from the hand maneuver, as reported by Miles [7]. Patients with LLN metastasis cannot be rescued with TME alone. LLA is necessary when LLN metastasis is present. Some patients with LLN metastasis can survive with TME+LLA, because LLN metastasis cannot be considered to be always a systemic disease.

On the other hand, one of the disadvantages from LLA is postoperative urinary or sexual functional disorder. The functional disorder after LLA is due to sacrificing the autonomic nerve plexus (TME+ LLA), which occurred at an extremely high rate during Period II, as reported by some authors [14,23]. Since 1985, autonomic nerve preservation (ANP) came into wide application for selected patients in our department. Functional disorder now has been reduced to a low rate, since ANP was applied in Period III (data not published), as also reported by many authors [43-46]. However, there are some important problems concerning ANP. When the nerve plexus is preserved, there is a risk in which cancer cells remain around the nerves. Another opinion is that cancer cells are unlikely to invade into the obturator space far away from the pelvic nerves without continuity. That is why, some surgeons say there is no meaning to perform LLA accompanied by ANP. But, our results have clearly shown better prognosis in patients who underwent ANP during Period III. Therefore, ANP seems to be not only reasonable to avoid functional disorder but also compatible with LLA, as reported by other authors $[16,27,31]$. Of course, the nerve plexus are sacrificed when cancer cells are suspected to have directly invaded themselves. ANP is selected depending upon tumor invasion, that means patients selection bias.
TME+LLA during Period III was performed only in patients with suspicious invasion to the nerve plexus. That is why, the disease-free survival in the TME+ LLA Group during Period III was extremely poorer than that in Period II, as shown in Fig. 5.

In order to establish the significance of LLA, a randomized controlled trial (RCT) may be needed. In fact, RCT has an advantage to avoid patient selection bias, but has an ethical disadvantage in perhaps not providing the most suitable treatment for each patient. In Japan, RCT is not ethically accepted. When LN metastases are accidentally found, we cannot ignore the LN metastases. At that time, we have desperately to perform lymphadenectomy, even though we had decided the unnecessity of lymphadenectomy before the operation. In such cases, RCT will fail. So, the clinicopathological data based on reliable long-term follow-up is extremely important. We can rescue some patients who have lateral LN metastasis, even though they are few in number. The optimal surgical treatment is that which is the most suitable for each individual patient, and the present study provides the essential support for such evidence-based medicine.

We concluded that TME and/or LLA was significant for reducing LR and improving survival in patients with Dukes C lower rectal cancer, compared to CVS. ANP was beneficial with LLA. TME+ANP was suitable for Dukes A or B disease.

\section{REFERENCES}

1. Gilchrist D, and David V. Prognosis in carcinoma of the bowel. Surg Gynecol Obstet 1948; 86:359-371.

2. Waugh J, and Kirklin J. The importance of the level of the lesion in the prognosis and treatment of carcinoma of the rectum and low sigmoid colon. Ann Surg 1949; 129:22-23.

3. Sauer I, and Bacon H. Influence of lateral spread of cancer of the rectum on radicality of operation and prognosis. Am J Surg 1951; 81:111-120.

4. Ault G, and Castro A. Clinical study of ligation of the inferior mesenteric artery in left colon resections. Surg Gynecol Obstet 1952; 94:223-228.

5. Ault G. A technique for cancer isolation and extended dissection for cancer of the distal colon and rectum. Surg Gynecol Obstet 1958; 106:467-477.

6. Miles E. A method of performing abdominopelvic excision for carcinoma of the rectum and of the terminal portion of the pelvic colon. Lancet 1908; 2:1812-1813.

7. Sauer I, and Bacon H. A new approach for excision of carcinoma of the lower portion of the rectum and anal canal. Surg Gynecol Obstet 1952; 95:229-242.

8. Sterns M, and Deddish M. Five-year results of abdominopelvic lymph node dissection for carcinoma of the rectum. Dis Colon Rectum 1959; 2:169-172. 
9. Harvey H, and Auchincloss H. Metastases to LNs from carcinomas that were arrested. Cancer 1968; 21:684-691.

10. Glass R, Ritche J, Thompson H, and Mann C. The results of surgical treatment of cancer of the rectum by radical resection and extended abdominoiliac lymphadenectomy. Br J Surg 1985; 72:599-601.

11. Enker WE, Pilipshen SJ, Heilweil ML, Sterns MW Jr., Janov AJ et al. En bloc pelvic lymphadenectomy and sphincter preservation in the surgical management of rectal cancer. Ann Surg 1986; 203:426-433.

12. Heald RJ, and Ryall RD. Recurrence and survival after total mesorectal excision for rectal cancer. Lancet 1986; $1: 1479-1482$.

13. Koyama Y, Moriya Y, and Hojo K. Effect of extended systemic lymphadenectomy for adenocarcinoma of the rectum - significant improvement of survival rate and decrease of LR. Jpn J Clin Oncol 1984; 14:623-632.

14. Hojo K, Sawada T, and Moriya Y. An analysis of survival and voiding sexual function after wide iliopelvic lymphadenectomy in patients with carcinoma of the rectum, compared with conventional lymphadenectomy. Dis Colon Rectum 1989; 32:128-133.

15. Moriya Y, Hojo K, Sawada T, and Koyama Y. Significance of lateral node dissection for advanced rectal carcinoma at or below the peritoneal reflection. Dis Colon Rectum 1989; 32:307-315.

16. Moriya Y, Sugihara K, Akasu T, and Fujita S. Importance of extended LN dissection for advanced lower rectal cancer. World J Surg 1997; 21:728-732.

17. Shirouzu K, Isomoto $H$, and Kakegawa T. A prospective clinicopathologic study of venous invasion in colorectal cancer. Am J Surg 1991; 162:216-222.

18. Shirouzu $\mathrm{K}$, Isomoto $\mathrm{H}$, and Kakegawa $\mathrm{T}$. Prognostic evaluation of perineural invasion in rectal cancer. Am J Surg 1993; 165:233-237.

19. Shirouzu K, Isomoto H, Morodomi T, and Kakegawa T. Carcinomatous lymphatic permeation. Prognostic significance in patients with rectal carcinoma - A long term prospective study. Cancer 1995; 75:4-10.

20. Shirouzu K, Isomoto $H$, and Kakegawa T. Distal spread of rectal cancer and optimal distal margin of resection for sphincter-preserving surgery. Cancer 1995; 76:388-392.

21. Kaplan EL, and Meier P. Non-parametric estimation from incomplete observation. J Am Stat Assoc 1958; 53:457481.

22. Peto R, Pike MC, Armitage NE, Breslow DR, Cox SV et al. Design and analysis of randomized clinical trials requiring prolonged observation of each patients. II. Analysis and examples. Br J Cancer 1977; 35:1-39.

23. Enker WE. Total mesorectal excision - the new golden standard of surgery for rectal cancer. Ann Med 1997; 29:127-133

24. Japanese research society for cancer of the colon and rectum. First English edn. Japanese classification of colorectal carcinoma, Kanehara \& Co. Ltd., Tokyo, 1997.

25. Pietra N, Sarli L, Costi R, Ouchemi C, Grattarola M et al. Role of follow-up in management of LR of colorectal cancer. A prospective randomized study. Dis Colon Rectum 1998; 41:1127-1133.
26. Blair JB, Holyoke EA, and Best RR. A note on the lymphatics of the middle and lower rectum and anus. Anat Rec 1950; 108:635-644.

27. Mori T, Takahashi K, and Yasuno M. Radical resection with autonomic nerve preservation and LN dissection techniques in lower rectal cancer surgery and its results: the impact of lateral LN dissection. Langenbecks Arch Surg 1999; 384:405-408

28. Takahashi T, Ueno M, Azekura K, and Ohta H. Lateral node dissection and total mesorectal excision for rectal cancer. Dis Colon Rectum 2000; 43(Suppl):S59-68.

29. Havenga K, Enker WE, Norstein J, Moriya Y, Heald RJ et al. Improved survival and local control after total mesorectal excision or D3 lymphadenectomy in the treatment of primary rectal cancer: An important analysis of 1411 patients. Eur J Surg Oncol 1999; 5:368-374.

30. Di Matteo G, Mascagni D, Lentini A, Tarroni D, and Filippini A. Advances in rectal cancer surgery. Dis Colon Rectum 1994; 37(Suppl):S50-53.

31. Di Matteo G, Mascagni D, Peparini N, and Di Matteo FM. Lymphadenectomy and nerve sparing technique in radical surgery of rectal cancer. Ann Ital Chir 1996; 67:593-602.

32. Hida J, Yasutomi M, Fujimoto K, Maruyama T, Okuno $\mathrm{K}$ et al. Does lateral $\mathrm{LN}$ dissection improve survival in rectal carcinoma? Examination of node metastases by the clearing method. J Am Coll Surg 1997; 184:475-480.

33. Fabrizio M, and Block GE. Morbidity and mortality of wide pelvic lymphadenectomy for rectal adenocarcinoma. Dis Colon Rectum 1992; 35:1143-1147.

34. Enker WE, Thaler HT, Cranor ML, and Polyak T. Total mesorectal excision in the operative treatment of carcinoma of the rectum. J Am Coll Surg 1995; 181:335346.

35. Horgan AF, and Finlay IG. Preoperative staging of rectal cancer allows selection of patients for preoperative radiotherapy. Br J Surg 2000; 87:575-579.

36. Bolognese A, Cardi M, Muttillo IA, Barbarosos A, Bocchetti $\mathrm{T}$ et al. Total mesorectal excision for surgical treatment of rectal cancer. J Surg Oncol 2000; 74:21-23.

37. Morodomi T, Isomoto H, Shirouzu K, Kakegawa T, Irie $\mathrm{K}$ et al. An index for estimating the probability of LN metastasis in rectal cancers. LN metastasis and histology of activity invasive regions of cancer. Cancer 1989; 63:539-543.

38. Sobin LH, and Wittekind C. International Union Against Cancer (UICC): TNM classification of malignant tumours, 5th ed., John Wiley \& Sons, New York, 1997.

39. Tada M, and Endo M. Ultrasonographic examination for lateral lymphatic spread and LR of rectal cancer: Preoperative detection and evaluation. Dis Colon Rectum 1995; 38:1047-1052.

40. Chiesura-Corona M, Muzzio PC, Giust G, Zuliani M, Pucciarelli $\mathrm{S}$ et al. Rectal cancer: CT local staging with histopathologic correlation. Abdom Imaging 2001; 26:134-138

41. Kim NK, Kim MJ, Park JK, Park SI, and Min JS. Preoperative staging of rectal cancer with MRI: accuracy and clinical usefulness. Ann Surg Oncol 2000; 7:732737. 
42. Pessaux P, Burtin P, and Arnaud JP. Staging for locoregional extension of rectal adenocarcinoma. Ann Chir 2001; 126:10-17.

43. Havenga $\mathrm{K}$, Enker WE, McDermott $\mathrm{K}$, Cohen AM, Minsky BD et al. Male and female sexual and urinary function after total mesorectal excision with autonomic nerve preservation for carcinoma of the rectum. J Am Coll Surg 1996; 182:495-502.

44. Masui H, Ike H, Yamaguchi S, Oki S, and Shimada H. Male sexual function after autonomic nerve preserving operation for rectal cancer. Dis Colon Rectum 1996; 39:1140-1145.

45. Sugihara K, Moriya Y, and Fujita S. Pelvic autonomic nerve preservation for patients with rectal carcinoma: Oncologic and functional outcome. Cancer 1996; 78:1871-1880.

46. Enker WE, Havenga K, Polyak T, Thaler H, and Cranor M. Abdominoperineal resection via total mesorectal excision and autonomic nerve preservation for low rectal cancer. World J Surg 1997; 21:715-720. 